BMJ Open

Diabetes

Research

\& Care

\title{
Determinants and correlates of adipose tissue insulin resistance index in Japanese women without diabetes and obesity
}

\author{
Kaori Kitaoka, ${ }^{1,2}$ Ayaka Tsuboi, ${ }^{1,3}$ Satomi Minato-Inokawa, ${ }^{1,4}$ Mari Honda, ${ }^{5,6}$ \\ Mika Takeuchi, ${ }^{1}$ Megumu Yano, ${ }^{1}$ Miki Kurata, ${ }^{1,7}$ Bin Wu, ${ }^{5,8}$ Tsutomu Kazumi (D) ,5,9,10 \\ Keisuke Fukuo ${ }^{1,5,7}$
}

To cite: Kitaoka K, Tsuboi A, Minato-Inokawa S, et al. Determinants and correlates of adipose tissue insulin resistance index in Japanese women without diabetes and obesity. BMJ Open Diab Res Care 2020;8:e001686. doi:10.1136/ bmjdrc-2020-001686

Received 13 June 2020 Revised 10 July 2020 Accepted 30 July 2020
Check for updates

(c) Author(s) (or their employer(s)) 2020. Re-use permitted under CC BY-NC. No commercial re-use. See rights and permissions. Published by BMJ.

For numbered affiliations see end of article.

Correspondence to Dr Tsutomu Kazumi; kazumi@mukogawa-u.ac.jp

\section{ABSTRACT}

Introduction Determinants and correlates of a novel index of adipose tissue insulin resistance (AT-IR) (the product of fasting insulin and free fatty acid concentrations) were investigated in Japanese women without diabetes and obesity.

Research design and methods Cross-sectional associations of AT-IR with fat mass and distribution, and IR-related cardiometabolic variables were examined in 210 young and 148 middle-aged women whose average body mass index (BMI) was $<23 \mathrm{~kg} / \mathrm{m}^{2}$ and waist was $<80 \mathrm{~cm}$. Multivariate linear regression analyses were used to identify most important determinants of AT-IR.

Results Young and middle-aged women did not differ in AT-IR (3.5 \pm 2.7 and 3.2 \pm 2.1 , respectively). In both young and middle-aged women, AT-IR was positively associated with trunk/leg fat ratio, a sophisticated measure of abdominal fat accumulation, fasting plasma glucose (FPG), fasting triglycerides (FTG), serum alanine aminotransferase and $\gamma$-glutamyl-transpeptidase (all $p<0.05$ ). Furthermore, in middle-aged but not in young women, AT-IR showed positive associations with BMI, waist, fat mass index, low-density lipoprotein cholesterol, apolipoprotein B and systolic and diastolic blood pressure (BP) (all $p<0.05$ ). AT-IR showed no association with hemoglobin A1c, highdensity lipoprotein (HDL) cholesterol and apolipoprotein A1 in two groups of women. On multivariate analysis including waist, FPG, FTG, HDL cholesterol and systolic BP as independent variables, FPG, FTG and HDL cholesterol emerged as independent determinants of AT-IR in young women (cumulative $R^{2}=0.141$ ) and waist in middle-aged women (cumulative $R^{2}=0.056$ ). In a model which included trunk/leg fat ratio instead of waist, trunk/leg fat ratio and systolic BP were determinants of AT-IR in middle-aged women (cumulative $R^{2}=0.093$ ). Results did not alter in young women.

Conclusions AT-IR may be a simple and useful surrogate index of adipose tissue insulin resistance even in populations without diabetes and obesity.

\section{INTRODUCTION}

Increased plasma concentrations of free fatty acid (FFA) are known to play an important role in the pathogenesis of insulin resistance

\section{Significance of this study}

What is already known about this subject?

- Adipose tissue insulin resistance (AT-IR) (the product of fasting insulin and free fatty acid concentrations) was less studied in populations without diabetes and obesity.

What are the new findings?

- Even in young and middle-aged Japanese women whose average body mass index was $<23 \mathrm{~kg} / \mathrm{m}^{2}$ and waist was $<80 \mathrm{~cm}$, AT-IR was associated positively with fasting glucose, fasting triglycerides and trunk/leg fat ratio, a sophisticated measure of abdominal fat accumulation.

- Furthermore, in middle-aged women, AT-IR showed positive associations with low-density lipoprotein cholesterol, apolipoprotein B and systolic blood pressure.

How might these results change the focus of research or clinical practice?

- AT-IR may be useful in assessing AT-IR even in women without diabetes and obesity.

(IR) of the muscle, liver and adipose tissue, a characteristic feature of type 2 diabetes. ${ }^{1}$ The important role of insulin in adipose tissue is to suppress triglyceride (TG) hydrolysis and release of FFA and glycerol into the circulation and to stimulate glucose uptake and TG synthesis. ${ }^{2}$ Excess adipose tissue FFA release resulting from increased lipolysis secondary to adipose tissue IR may induce or aggravate IR in muscle and liver in obesity and type 2 diabetes. $^{3}$

Unfortunately, no consensus exists on how to determine adipose tissue insulin sensitivity. ${ }^{45}$ The gold standard to measure adipose tissue IR is a hyperinsulinemic-euglycemic clamp procedure in conjunction with a fatty acid tracer infusion. ${ }^{6}$ However, owing to 
feasibility, cost and labor intensiveness, this procedure limits the number of subjects and hence large-scale studies are difficult.

One potential method to investigate adipose tissue IR is a novel index of the adipose tissue insulin resistance (AT-IR), which is calculated by the product of fasting concentrations of insulin and FFAs ${ }^{457}$ and is equivalent to the homeostatic model assessment of insulin resistance (HOMA-IR) index. ${ }^{8}$ This index has been validated using the gold standard to measure AT-IR, a hyperinsulinemiceuglycemic clamp procedure in conjunction with a fatty acid tracer infusion. ${ }^{45}$ AT-IR has been shown to be associated with hepatic fat content, a marker of ectopic fat deposition, in patients with non-alcoholic steatohepatitis. ${ }^{9}$ It has been shown that AT-IR was associated with obesity, metabolic syndrome, prediabetes and type 2 diabetes. ${ }^{10-16}$ However, most studies which employed AT-IR investigated people with obesity and/or type 2 diabetes and were conducted in Western countries. ${ }^{10-16}$ We, therefore, investigated determinants, correlates and usefulness of AT-IR in Japanese women without diabetes and obesity, populations in which confounding factors are so scarce. ${ }^{17}$ Because we have shown that endurance training lowered AT-IR in young women ${ }^{18}$ we excluded female collegiate athletes in the present study.

\section{SUBJECTS AND METHODS}

We cross-sectionally studied 210 young and 148 middleaged Japanese women whose details were reported elsewhere. ${ }^{17-21}$ Young women were female Japanese students of Department of Food Sciences and Nutrition, Mukogawa Women's University and middle-aged women were the biological mothers of 148 students who participated in the study. Subjects with clinically diagnosed acute or chronic inflammatory diseases, endocrine, cardiovascular, hepatic, renal diseases, hormonal contraception, unusual dietary habits were excluded from the study.

After a 12-hour overnight fast, blood samples were obtained and blood pressure (BP) and anthropometric index were measured. Systolic and diastolic BP were measured using a fully automated sphygmomanometer (BP-203RV II, Colin, Tokyo, Japan) after participants had rested at least $5 \mathrm{~min}$. Plasma glucose (PG) was determined by the hexokinase/glucose-6-phosphate dehydrogenase method (interassay coefficient of variation $(\mathrm{CV})<2 \%$ ). Serum insulin was measured by an ELISA method with a narrow specificity excluding des-31, des-32 and intact proinsulin (interassay CV $<6 \%$ ). Serum TG, cholesterol, high-density lipoprotein (HDL) cholesterol, apolipoprotein AI (apoAI) and apolipoprotein B-100 (apoB), FFA, aspartate aminotransferase (AST), alanine aminotransferase (ALT) and $\gamma$-glutamyl-transpeptidase (GGT) were measured as previously reported. ${ }^{17-19}$ Low-density lipoprotein (LDL) cholesterol was calculated using the Friedewald's formula. HOMA-IR was calculated as a product of fasting PG and insulin levels. ${ }^{8}$ AT-IR was calculated as a product of fasting concentrations of insulin $(\mu \mathrm{U} / \mathrm{mL})$ and FFA $(\mathrm{mEq} / \mathrm{L}) \cdot{ }^{45}$ Metabolic syndrome was defined according to the modified criteria of the National Cholesterol Education Program Adult Treatment Panel III guidelines with the Japanese definition of abdominal obesity. ${ }^{22}$

Whole-body dual-energy X-ray absorptiometry (DXA) (Hologic QDR-2000 software V.7.20D, Waltham, Massachusetts, USA) was used to measure lean tissue mass, fat mass and bone mineral mass for arms, legs (lower body), trunk and the total body. ${ }^{17}$ General adiposity was assessed using body mass index (BMI) and fat mass index (FMI), the latter of which was calculated as body fat mass in $\mathrm{kg}$ divided by height in meter squared. Waist circumference and the ratio of trunk to leg fat ${ }^{23}$ were considered markers of abdominal fat accumulation.

Data were presented as mean \pm SD unless otherwise stated. Due to deviation from normal distribution, liver enzymes were logarithmically transformed for analysis. Bivariate correlations of AT-IR with cardiometabolic parameters were evaluated by Pearson's correlation analysis. Stepwise multiple linear regression analyses were performed to further identify the most significant variables contributing to the variation of AT-IR. A two-tailed $\mathrm{p}<0.05$ was considered to be statistically significant. All calculations were performed with SPSS system V.15.0 (SPSS, Chicago, Illinois, USA).

\section{RESULTS}

On average, 210 young and 148 middle-aged women were normoglycemic, normolipidemic and normotensive (table 1). Their serum liver enzyme concentrations were normal as well. Middle-aged compared with young women had higher BMI, waist circumference, FMI and trunk/leg fat ratio. Fasting PG was higher, FFA was somewhat higher but fasting insulin tended to be lower in middle-aged compared with young women. Therefore, AT-IR and HOMA-IR did not differ in two age groups (table 1). Serum apoAI and HDL cholesterol were slightly but significantly higher in middle-aged compared with young women even in the presence of higher TG in the former. LDL cholesterol, apoB, liver enzyme concentrations and $\mathrm{BP}$ all were higher in middle-aged women.

Among 118 young non-athlete women and 65 middle-aged women who underwent a standard 75 $\mathrm{g}$ oral glucose tolerance test, ${ }^{20} 217$ young and 14 middle-aged women had prediabetes diagnosed based on glycemia criteria (fasting and 2-hour PG) of the American Diabetes Association. ${ }^{24}$ AT-IR tended to be higher in middle-aged women with prediabetes compared with those with normal glucose tolerance $(3.7 \pm 2.5$ vs $2.4 \pm 1.4, \mathrm{p}=0.06$, figure 1$)$, although HOMA-IR did not differ ( $1.5 \pm 1.1$ vs $0.9 \pm 0.5, \mathrm{p}=0.11)$. In young women, AT-IR $(4.1 \pm 3.0$ vs $2.8 \pm 2.0, \mathrm{p}=0.12)$ and HOMA-IR $(1.5 \pm 1.3$ vs $1.2 \pm 0.6, \mathrm{p}=0.23)$ did not differ between prediabetes and normal glucose tolerance. We could not compare AT-IR in women with 
Table 1 Anthropometric and cardiometabolic features of young and middle-aged Japanese women

\begin{tabular}{|c|c|c|c|}
\hline & Young & Middle-aged & \\
\hline & $\mathrm{n}=\mathbf{2 1 0}$ & $n=148$ & $P$ value \\
\hline AT-IR & $3.5 \pm 2.7$ & $3.2 \pm 2.1$ & 0.417 \\
\hline Age (years) & $20.7 \pm 1.2$ & $49.8 \pm 3.6$ & $<0.001$ \\
\hline Body mass index $\left(\mathrm{kg} / \mathrm{m}^{2}\right)$ & $20.3 \pm 2.3$ & $22.0 \pm 2.8$ & $<0.001$ \\
\hline Waist circumference (cm) & $71.2 \pm 5.7$ & $78.7 \pm 8.1$ & $<0.001$ \\
\hline Fat mass index $\left(\mathrm{kg} / \mathrm{m}^{2}\right)$ & $5.5 \pm 1.7$ & $6.7 \pm 2.4$ & $<0.001$ \\
\hline Trunk/leg fat ratio & $1.25 \pm 0.25$ & $1.64 \pm 0.39$ & $<0.001$ \\
\hline Fasting glucose (mg/dL) & $82 \pm 7$ & $89 \pm 14$ & $<0.001$ \\
\hline Fasting insulin ( $\mu \mathrm{U} / \mathrm{mL})$ & $6.0 \pm 3.1$ & $5.4 \pm 2.8$ & 0.070 \\
\hline Fasting FFA (mEq/L) & $0.56 \pm 0.22$ & $0.60 \pm 0.23$ & 0.118 \\
\hline HbA1c (\%) & $5.2 \pm 0.2$ & $5.5 \pm 0.4$ & $<0.001$ \\
\hline HOMA-IR & $1.22 \pm 0.82$ & $1.21 \pm 0.71$ & 0.896 \\
\hline Triglyceride (mg/dL) & $58 \pm 38$ & $81 \pm 36$ & $<0.001$ \\
\hline Cholesterol (mg/dL) & $182 \pm 29$ & $224 \pm 35$ & $<0.001$ \\
\hline HDL cholesterol (mg/dL) & $74 \pm 13$ & $77 \pm 16$ & 0.030 \\
\hline $\begin{array}{l}\text { Non-HDL cholesterol } \\
(\mathrm{mg} / \mathrm{dL})\end{array}$ & $108 \pm 25$ & $147 \pm 33$ & $<0.001$ \\
\hline LDL cholesterol (mg/dL) & $97 \pm 25$ & $130 \pm 30$ & $<0.001$ \\
\hline $\begin{array}{l}\text { Apolipoprotein A1 } \\
(\mathrm{mg} / \mathrm{dL})\end{array}$ & $164 \pm 20$ & $178 \pm 22$ & $<0.001$ \\
\hline Apolipoprotein B (mg/dL) & $68 \pm 15$ & $93 \pm 19$ & $<0.001$ \\
\hline AST $(U / L)^{*}$ & $18 \pm 5$ & $21 \pm 13$ & 0.003 \\
\hline $\operatorname{ALT}(\mathrm{U} / \mathrm{L})^{*}$ & $13 \pm 6$ & $20 \pm 18$ & $<0.001$ \\
\hline GGT (U/L)* & $14 \pm 4$ & $22 \pm 20$ & $<0.001$ \\
\hline $\begin{array}{l}\text { Systolic blood pressure } \\
(\mathrm{mm} \mathrm{Hg})\end{array}$ & $108 \pm 11$ & $121 \pm 16$ & $<0.001$ \\
\hline $\begin{array}{l}\text { Diastolic blood pressure } \\
(\mathrm{mm} \mathrm{Hg})\end{array}$ & $63 \pm 8$ & $74 \pm 11$ & $<0.001$ \\
\hline
\end{tabular}

Mean + SD

*logarithmically transformed for analyses.

ALT, alanine aminotransferase; AST, aspartate aminotransferase; AT-IR, adipose tissue insulin resistance; FFA, free fatty acid; GGT, $\gamma$-glutamyl-transpeptidase; HbA1c, hemoglobin A1c; HDL, high-density lipoprotein; HOMA-IR, homeostatic model assessment of insulin resistance; LDL, low-density lipoprotein.

and without metabolic syndrome due to the small number of women with metabolic syndrome (three middle-aged women in total). However, AT-IR was higher in middle-aged women with BMI $\geq 25 \mathrm{~kg} / \mathrm{m}^{2}$ $(\mathrm{n}=22)$ compared with underweight women $(\mathrm{n}=10)$ $(4.3 \pm 2.4$ vs $2.5 \pm 1.2, \mathrm{p}<0.05)$ (figure 1 ) but not in young women (data not shown).

AT-IR strongly associated with HOMA-IR both in young and middle-aged women (table 2). In young women, AT-IR positively associated with trunk/leg fat ratio. In addition, fasting PG and fasting TG positively associated with AT-IR as well. Furthermore, ALT and GGT showed positive, although modest, associations with AT-IR. These associations were confirmed in middle-aged women. In addition, AT-IR showed positive associations with BMI,

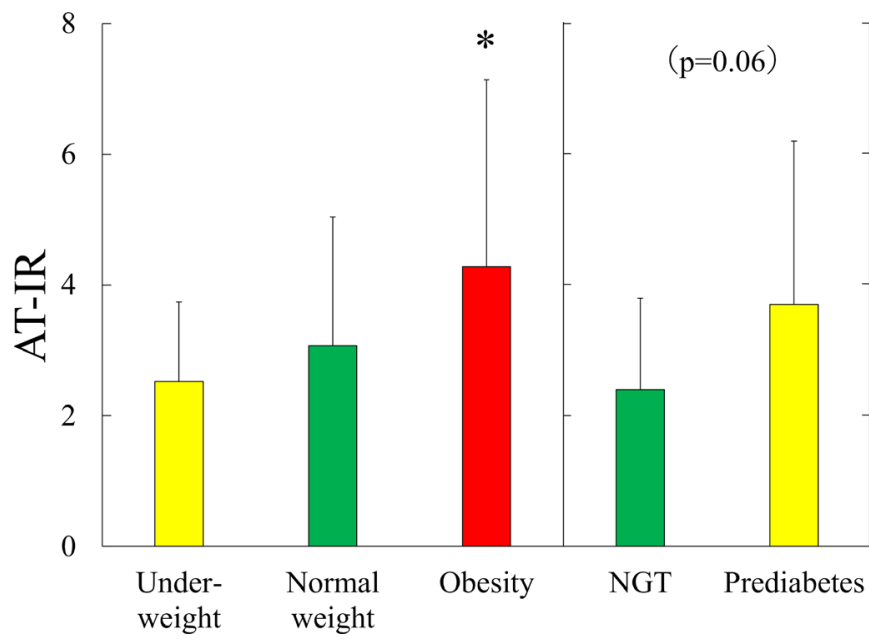

Figure 1 Adipose tissue insulin resistance index (AT-IR) in middle-aged women. Mean $\pm S D$. Left panel: underweight ( $n=10$, yellow column), normal weight ( $n=112$, green column) and middle-aged women with obesity ( $n=22$, red column). Analysis of variance; $p=0.020$. ${ }^{*} P<0.05$ versus underweight by Bonferroni's multiple comparison procedure. Right panel: women with normal glucose tolerance (NGT, $n=51$, green column) and prediabetes ( $n=14$, yellow column).

waist circumference and FMI, and with LDL cholesterol, apoB and systolic and diastolic BP in middle-aged but not in young women. There was no association with HDL cholesterol and apoA1 in two groups of women.

We have done multivariate analysis for AT-IR as a dependent variable. In the model including five diagnostic components of metabolic syndrome as independent variables, fasting $\mathrm{PG}$, fasting $\mathrm{TG}$ and $\mathrm{HDL}$ cholesterol emerged as significant determinants of AT-IR in young women independently of waist circumference and systolic BP. These three variables explained $14.1 \%$ of the variation in AT-IR in young women. In middle-aged women, waist circumference was the only determinant of AT-IR, accounting for $5.6 \%$ of the variation. When trunk/ leg fat ratio was included instead of waist circumference, trunk/leg fat ratio and systolic BP emerged as independent determinants of AT-IR in middle-aged women and explained $9.3 \%$ of AT-IR variability. Results of young women did not alter.

\section{DISCUSSION}

The current study has demonstrated that lean Japanese women in early 20 s showed positive associations of AT-IR, a novel index of adipose tissue insulin resistance, with trunk/leg fat ratio, fasting PG and TG, ALT and GGT. Among these variables, fasting PG, fasting TG and HDL cholesterol were independent determinants of AT-IR. In middle-aged Japanese women without diabetes, association between AT-IR and obesity and prediabetes, although marginally significant $(\mathrm{p}=0.06)$, was confirmed as previously reported in adolescents, ${ }^{12} 15$ middle-aged and elderly people with obesity. ${ }^{10131416}$ AT-IR also showed positive 
Table 2 Correlation analysis between AT-IR and anthropometric and cardiometabolic variables in young and middle-aged Japanese women

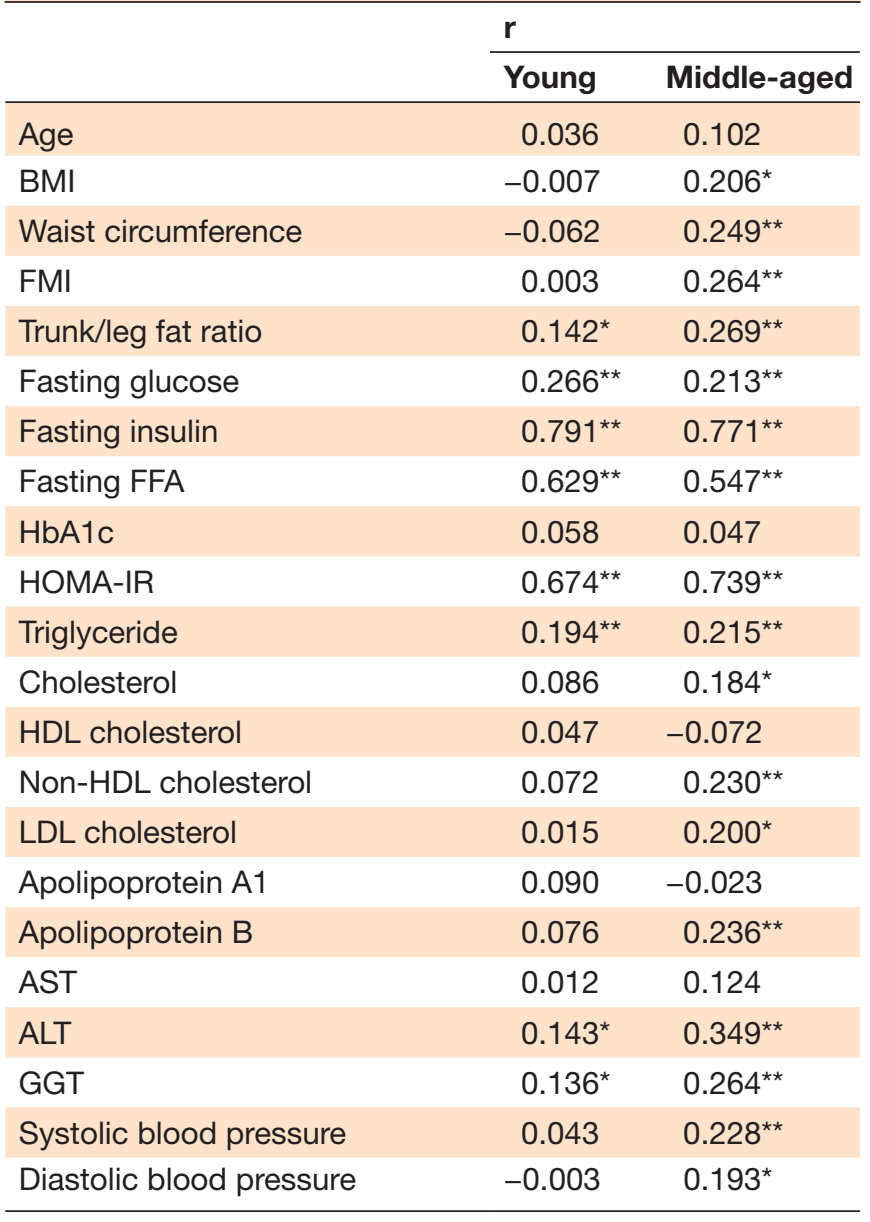

Mean \pm SD.

${ }^{*} \mathrm{P}<0.05,{ }^{* *} \mathrm{p}<0.01$ or less.

ALT, alanine aminotransferase; AST, aspartate aminotransferase; AT-IR, adipose tissue insulin resistance; BMI, body mass index; FFA, free fatty acid; FMI, fat mass index; GGT, $\gamma$-glutamyltranspeptidase; $\mathrm{HbA1c}$, hemoglobin A1c; HDL, high-density lipoprotein; HOMA-IR, homeostatic model assessment of insulin resistance; LDL, low-density lipoprotein.

associations with a broad range of cardiometabolic risk factors, including all diagnostic components of metabolic syndrome. Among those variables, central or abdominal fat accumulation (waist circumference and trunk/leg fat ratio) was an independent determinant of AT-IR in middle-aged Japanese women without diabetes. These findings suggest that AT-IR may be a simple and useful surrogate index of adipose tissue insulin resistance.

In the present study, AT-IR associated with waist circumference, a better predictor of diabetes risk for Asian women, ${ }^{25}$ and an essential component for the diagnosis of metabolic syndrome in Japan, ${ }^{26}$ in middle-aged Japanese women but not in young women. However, young women showed significant association with trunk/leg fat ratio, a sophisticated measure of abdominal fat accumulation. ${ }^{23}$ A recent study in middle-aged Japanese men without diabetes and obesity has demonstrated that moderate abdominal subcutaneous fat accumulation was associated with reduced adipose tissue insulin sensitivity. ${ }^{27}$ Because trunk fat consists of abdominal subcutaneous and visceral fat, these findings may indicate a pathophysiological importance of central fat accumulation in adipose tissue insulin sensitivity in lean Japanese women in early 20 s and in middle-aged Japanese men and women without diabetes and obesity and suggest the usefulness of AT-IR in epidemiological and clinical studies.

Association between adipose IR assessed by AT-IR and prediabetes has previously been reported in adolescents, ${ }^{13}{ }^{15}$ middle-aged and elderly people with obesity $^{11} 14^{16}$ and confirmed in middle-aged Japanese women without diabetes, although marginally significant $(\mathrm{p}=0.06)$, in the present study. Furthermore, direct association of AT-IR with fasting PG and fasting TG was evident even in lean Japanese women in early 20s whose fasting PG averaged $82 \mathrm{mg} / \mathrm{dL}$ and fasting TG $58 \mathrm{mg} /$ dL. These findings suggest that adipose IR may be associated with the worsening of fasting PG and TG within respective normal reference range. In contrast, there was no association of AT-IR with HDL cholesterol and apoA1, major apolipoprotein of HDL particles, in two age groups of women whereas inverse correlation between AT-IR and HDL cholesterol was evident in youth with obesity. ${ }^{15}$ This discrepancy may be due in part to high HDL cholesterol, which is accounted for by genetic deficiency of cholesteryl ester transfer protein, in Japanese people. ${ }^{28}$ Indeed, HDL cholesterol averaged $77 \mathrm{mg} / \mathrm{dL}$ in middle-aged Japanese women while it was $49 \mathrm{mg} / \mathrm{dL}$ in normal weight youth. ${ }^{15}$ However, it is noted that HDL cholesterol emerged as an independent determinant of AT-IR in young women in the present study.

Pont $e t a l^{29}$ investigated a stable isotope kinetic study of apoB-containing lipoproteins in insulin-resistant women with abdominal obesity, normal fasting triglyceridemia and normal glucose tolerance. They found an increased production rate of apoB in very low density lipoprotein by the liver, leading to significantly higher apoB concentrations, suggesting a hepatic resistance to the inhibitory effect of insulin on very low density lipoprotein apoB production at an early stage of IR linked with abdominal obesity. ${ }^{29}$ In the present study, middle-aged compared with young women had higher waist circumference and apoB, although only 3 of 148 middle-aged women met the criteria for metabolic syndrome. AT-IR correlated with apoB, non-HDL and LDL cholesterol in middleaged but not in young women. These findings suggest that at a very early stage of IR in the absence of abdominal obesity, adipose IR may be associated in part with increased serum number of apoB-containing lipoprotein particles, which are highly atherogenic. ${ }^{30}$

Stronger associations among biomarkers of metabolic syndrome in women with obesity compared with lean women ${ }^{31}$ may be in line with our observation that associations of AT-IR with ALT, GGT and BP were stronger in middle-aged compared with young women. 
Higher average AT-IR, although not significant, in young compared with middle-aged women was due to higher fasting insulin $(\mathrm{p}=0.07)$ in young women despite lower BMI and waist circumference. However, results of table 1 other than AT-IR, fasting insulin and HOMA-IR clearly showed that insulin sensitivity is superior in young compared with middle-aged women, who were not in insulin-resistant condition as their HOMA-IR averaged 1.2. These observations suggest that effects of adiposity on fasting insulin concentrations may not be as strong in insulin-sensitive subjects without diabetes and obesity as in insulin-resistant subjects with obesity.

The homogeneous study population with few confounding factors ${ }^{17}$ and the accurate and reliable measures of general and central fat accumulation by DXA are the strength of the present study. Several limitations of this study include the cross-sectional design, relatively small sample size and a single measurement of biochemical variables. Statistical power was not calculated. As participants were young and middle-aged Japanese women, the results may not be generalized to other gender, age populations, races or ethnicities.

In conclusion, AT-IR associated with a broad range of cardiometabolic risk factors, including all diagnostic components of metabolic syndrome, in healthy Japanese women without diabetes. AT-IR is a simple surrogate index of adipose tissue insulin resistance, a key pathophysiological component of type 2 diabetes, which can be used in large-scale clinical and epidemiological studies.

\section{Author affiliations \\ ${ }^{1}$ Research Institute for Nutrition Sciences, Mukogawa Women's University, Nishinomiya, Japan \\ ${ }^{2}$ Department of Public Health, Shiga University of Medical Science, Otsu, Japan \\ ${ }^{3}$ Department of Nutrition, Osaka City Juso Hospital, Osaka, Japan \\ ${ }^{4}$ Laboratory of Community Health and Nutrition, Department of Bioscience, Graduate School of Agriculture, Ehime University, Matsuyama, Japan \\ ${ }^{5}$ Open Research Center for Studying of Lifestyle-Related Diseases, Mukogawa \\ Women's University, Nishinomiya, Japan \\ ${ }^{6}$ Department of Health, Sports, and Nutrition, Faculty of Health and Welfare, Kobe Women's University, Kobe, Japan \\ ${ }^{7}$ Department of Food Sciences and Nutrition, Mukogawa Women's University, Nishinomiya, Japan \\ ${ }^{8}$ Department of Endocrinology, First Affiliated Hospital of Kunming Medical University, Kunming, China \\ ${ }^{9}$ Mukogawa Joshi Daigaku Eiyo kagakukenkyujo nitsu, Nishinomiya, Japan \\ ${ }^{10}$ Department of Medicine, Kohnan Kakogawa Hospital, Kakogawa, Japan}

Acknowledgements The authors would like to thank all participants for their dedicated and conscientious collaboration.

Contributors KK, AT, SM-I, MH, MT, MY, MK and BW collected and analyzed data. TK wrote the manuscript and KF reviewed and edited it. All authors approved the final version of the manuscript to be published. TK supervised the study, had full access to all the data in the study and takes responsibility for the integrity of the data and the accuracy of the data analysis.

Funding The authors have not declared a specific grant for this research from any funding agency in the public, commercial or not-for-profit sectors.

Competing interests None declared.

Patient consent for publication Not required.

Ethics approval The study was approved by the Ethical Committees of Mukogawa Women's University (NNo. 07-28, on 19 February 2008) and was in accordance with the tenets of the Declaration of Helsinki. All subjects were recruited as volunteers and gave written consent after the experimental procedure had been explained.

Provenance and peer review Not commissioned; externally peer reviewed.

Data availability statement. All data relevant to the study are included in the article. The ethical committee of the University does not allow us to open data except for a manuscript.

Open access This is an open access article distributed in accordance with the Creative Commons Attribution Non Commercial (CC BY-NC 4.0) license, which permits others to distribute, remix, adapt, build upon this work non-commercially, and license their derivative works on different terms, provided the original work is properly cited, appropriate credit is given, any changes made indicated, and the use is non-commercial. See: http://creativecommons.org/licenses/by-nc/4.0/.

ORCID iD

Tsutomu Kazumi http://orcid.org/0000-0002-8855-9268

\section{REFERENCES}

1 Bays H, Mandarino L, DeFronzo RA. Role of the adipocyte, free fatty acids, and ectopic fat in pathogenesis of type 2 diabetes mellitus: peroxisomal proliferator-activated receptor agonists provide a rational therapeutic approach. $J$ Clin Endocrinol Metab 2004;89:463-78.

2 Saponaro C, Gaggini M, Carli F, et al. The subtle balance between lipolysis and lipogenesis: a critical point in metabolic homeostasis. Nutrients 2015;7:9453-74.

3 Delarue J, Magnan C. Free fatty acids and insulin resistance. Curr Opin Clin Nutr Metab Care 2007;10:142-8.

4 Søndergaard E, Jensen MD. Quantification of adipose tissue insulin sensitivity. J Investig Med 2016;64:989-91.

5 Søndergaard E, Espinosa De Ycaza AE, Morgan-Bathke M, et al. How to measure adipose tissue insulin sensitivity. J Clin Endocrinol Metab 2017;102:1193-9.

6 Jensen MD, Nielsen S. Insulin dose response analysis of free fatty acid kinetics. Metabolism 2007;56:68-76.

7 ter Horst KW, van Galen KA, Gilijamse PW, et al. Methods for quantifying adipose tissue insulin resistance in overweight/obese humans. Int J Obes 2017;41:1288-94.

8 Matthews DR, Hosker JP, Rudenski AS, et al. Homeostasis model assessment: insulin resistance and beta-cell function from fasting plasma glucose and insulin concentrations in man. Diabetologia 1985;28:412-9.

9 Gastaldelli A, Harrison SA, Belfort-Aguilar R, et al. Importance of changes in adipose tissue insulin resistance to histological response during thiazolidinedione treatment of patients with nonalcoholic steatohepatitis. Hepatology 2009;50:1087-93.

10 Abdul-Ghani MA, Molina-Carrion M, Jani R, et al. Adipocytes in subjects with impaired fasting glucose and impaired glucose tolerance are resistant to the anti-lipolytic effect of insulin. Acta Diabetol 2008;45:147-50.

11 Adams-Huet B, Devaraj S, Siegel D, et al. Increased adipose tissue insulin resistance in metabolic syndrome: relationship to circulating adipokines. Metab Syndr Relat Disord 2014;12:503-7.

12 Hershkop K, Besor O, Santoro N, et al. Adipose insulin resistance in obese adolescents across the spectrum of glucose tolerance. $J$ Clin Endocrinol Metab 2016;101:2423-31.

13 Gastaldelli A, Gaggini M, DeFronzo RA. Role of adipose tissue insulin resistance in the natural history of type 2 diabetes: results from the San Antonio metabolism study. Diabetes 2017;66:815-22.

$14 \mathrm{Kim}$ JY, Bacha F, Tfayli H, et al. Adipose tissue insulin resistance in youth on the spectrum from normal weight to obese and from normal glucose tolerance to impaired glucose tolerance to type 2 diabetes. Diabetes Care 2019;42:265-72.

15 Hagman E, Besor O, Hershkop K, et al. Relation of the degree of obesity in childhood to adipose tissue insulin resistance. Acta Diabetol 2019;56:219-26.

16 Cai X, Xia L, Pan Y, et al. Differential role of insulin resistance and $\beta$-cell function in the development of prediabetes and diabetes in middle-aged and elderly Chinese population. Diabetol Metab Syndr 2019;11:24.

17 Tanaka M, Yoshida T, Bin W, et al. FTO, abdominal adiposity, fasting hyperglycemia associated with elevated HbA1c in Japanese middleaged women. J Atheroscler Thromb 2012;19:633-42.

18 Kitaoka K, Takeuchi M, Tsuboi A, et al. Increased adipose and muscle insulin sensitivity without changes in serum adiponectin in young female collegiate athletes. Metab Syndr Relat Disord 2017;15:246-51. 
19 Terazawa-Watanabe M, Tsuboi A, Fukuo K, et al. Association of adiponectin with serum preheparin lipoprotein lipase mass in women independent of fat mass and distribution, insulin resistance, and inflammation. Metab Syndr Relat Disord 2014;12:416-21.

20 Tsuboi A, Minato S, Yano M, et al. Association of serum orosomucoid with 30-min plasma glucose and glucose excursion during oral glucose tolerance tests in non-obese young Japanese women. BMJ Open Diabetes Res Care 2018;6:e000508.

21 Tsuboi A, Kitaoka K, Yano M, et al. Higher circulating orosomucoid and lower early-phase insulin secretion in midlife Japanese with slower glucose disposal during oral glucose tolerance tests. Diabetol Int 2020;11:27-32.

22 Alberti KGMM, Eckel RH, Grundy SM, et al. Harmonizing the metabolic syndrome: a joint interim statement of the International diabetes Federation Task force on epidemiology and prevention; National heart, lung, and blood Institute; American heart association; world heart Federation; international atherosclerosis Society; and international association for the study of obesity. Circulation 2009;120:1640-5.

23 Lim U, Turner SD, Franke AA, et al. Predicting total, abdominal, visceral and hepatic adiposity with circulating biomarkers in Caucasian and Japanese American women. PLoS One 2012;7:e43502.
24 American Diabetes Association. 2. Classification and Diagnosis of Diabetes: Standards of Medical Care in Diabetes-2018. Diabetes Care 2018;41:S13-27.

25 Luo J, Hendryx M, Laddu D, et al. Racial and ethnic differences in anthropometric measures as risk factors for diabetes. Diabetes Care 2019;42:126-33.

26 Metabolic Syndrome Criteria Study Group. Definition and criteria for metabolic syndrome. J Jpn Soc Intern Med 2005;94:188-203. (in Japanese).

27 Sugimoto D, Tamura Y, Takeno K, et al. Clinical features of nonobese, apparently healthy, Japanese men with reduced adipose tissue insulin sensitivity. J Clin Endocrinol Metab 2019;104:2325-33.

28 Yokoyama S. Unique features of high-density lipoproteins in the Japanese: in population and in genetic factors. Nutrients 2015;7:2359-81.

29 Pont F, Duvillard L, Florentin E, et al. Early kinetic abnormalities of apoB-containing lipoproteins in insulin-resistant women with abdominal obesity. Arterioscler Thromb Vasc Biol 2002;22:1726-32.

30 Sniderman AD, Thanassoulis G, Glavinovic T, et al. Apolipoprotein $B$ particles and cardiovascular disease: a narrative review. JAMA Cardiol 2019;4:1287-95.

31 Abdullah AR, Hasan HA, Raigangar VL. Analysis of the relationship of leptin, high-sensitivity C-reactive protein, adiponectin, insulin, and uric acid to metabolic syndrome in lean, overweight, and obese young females. Metab Syndr Relat Disord 2009;7:17-22. 